\title{
A Time-Reduced Method for Calculation Distortions in Envelope Tracking Power Amplifiers
}

\author{
A. O. Pergushev ${ }^{1}$, V. A. Sorotsky ${ }^{2}$ \\ Peter the Great St. Petersburg Polytechnic University, \\ St. Petersburg, Russian Federation \\ E-mail: ${ }^{1}$ pergushev.aleksey@mail.ru, ${ }^{2}$ sorotsky@mail.spbstu.ru
}

Received: June 20, 2018

\begin{abstract}
To increase the efficiency of power amplifiers in wireless telecommunications systems, whose signals have a high peak to average power ratio, the envelope tracking method can be used. This method involves forming of a low-frequency envelope signal by a switched-mode power amplifier (SPA), which is then used as a supply voltage for a RF power amplifier. A specific feature of the SPA is that its output signal has high level of harmonic distortions. To evaluate these harmonic distortions, computer simulation can be used. However, time required to perform one cycle of simulation can be as much as several hours because of high complexity of SPA models. To reduce the simulation time of signal spectrum, approximate method that allows to represent the output voltage of a SPA in analytical form can be used. However, in known publications, the non-zero switching time of transistors is not taken into consideration, which leads to an appreciable error in the determination of the spectral components. This paper presents a method of analytic calculation of SPA output signal spectrum that considers the linear and exponential approximations of non-zero switching times of transistor. It allows to decrease the error in determining the harmonic distortions from $30 \ldots 35 \mathrm{~dB}$ to $1 \ldots 6 \mathrm{~dB}$ and to reduce the time expenditure as compared with computer simulation as much as 300 through 800 times.
\end{abstract}

Keywords: Envelope tracking, multiphase switched-mode power amplifier, approximate analytic method, computer simulation, harmonic distortions, time expenditure reduction.

\section{INTRODUCTION}

The signals being applied in wireless telecommunication systems (LTE, W-CDMA, etc.) have rather high peak to average power ratio. To increase efficiency of power amplifiers of such signals the envelope tracking method can be used. This method assumes forming low frequency envelope signal with switched-mode power amplifier (SPA). In that case, SPA should not make any appreciable distortions in this low-frequency signal. As is known, to reduce distortions, it is expedient to use multiphase SPA [1,6] in which intermodulation products of a reference voltage and an envelope signal can be shifted to the point on the frequency axis determined by the product $N q$ where $N$ - quantity of the SPA phases, $q$ - the ratio of the reference voltage frequency to the envelope signal frequency. It allows to lower requirements to the low-pass filter (LPF) by means of which attenuation of the harmonic distortion and intermodulation components in output SPA signal is carried out. The Figure 1 represents an example of a test signal $(a)$, a reference voltage for a four-phase SPA $(b)$ and the output voltage of the SPA $(c)$.

(C) Pergushev A. O., Sorotsky V. A., 2018 


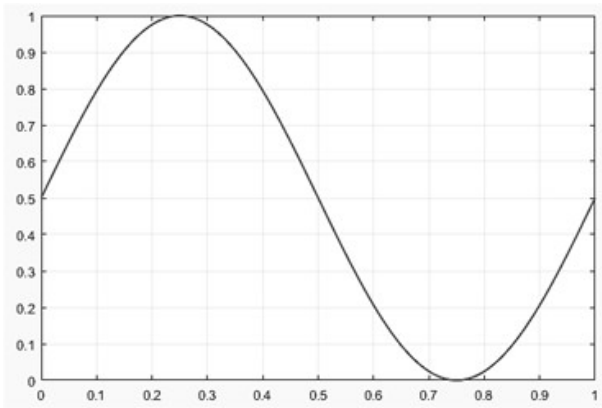

(a)

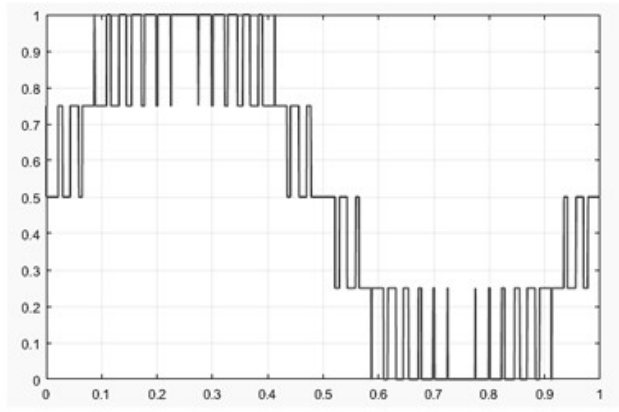

(c)

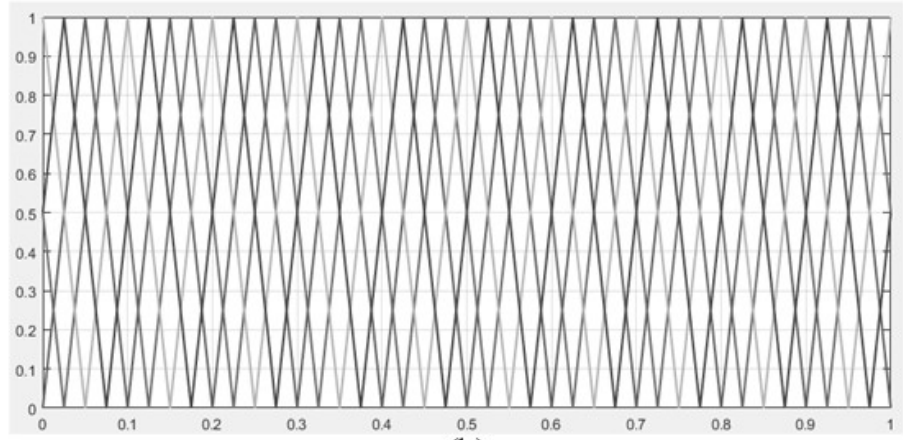

(b)

Figure 1. The test signal $(a)$, the reference voltage of the four-phase SPA $(b)$ and the output voltage of the SPA $(c)$ - in relative units

The most significant problem arises due to harmonic distortions in SPA, which can get to the LPF bandwidth.

In [2-5], where the principles of SPA implementation are considered, the problem of envelope signal harmonic distortions caused by imperfect features of transistors (non-zero switching time, forward on voltage drop, influence of parasitic inductance of outputs of transistors and elements of the printed circuit board, etc.) isn't raised. Influence of the said parameters can be considered by means of computer simulation. It is favored by that many companies provide the tested simulation models of their electronic devices. Unfortunately, as it was revealed in practice, the total time expenditure for simulation in case of different combinations of the varied parameters can reach noticeable value (tens of minutes through hours) as the SPA model usually include dozens of transistors.

The replacement of transistors' complex models with idealized switches of course allows to lower time expenditure, but at the same time simulation accuracy significantly reduces. It can be seen on the spectral diagrams in Figure $3(a),(c),(e)$ and Figure $4(a),(c),(e)$, which represent the harmonic distortions amplitudes calculated using the SPA simulation model (dark tone histogram) and similar characteristics obtained on the assumption that the transistors have zero switching times (light-tone histogram). These spectral diagrams correspond to $q=10$ and $N=4(a), 6(c)$ and $8(e)$. The abscissas of the diagrams indicate the harmonics' number.

The error in determining the amplitudes of the harmonic distortion components which in the diagrams are located to the left of a set of intermodulation products (its position is determined by the product of $q N$ ) reaches $30 \ldots 35 \mathrm{~dB}$. In addition, in order to assess the effect of the transistors' none-zero switching times on the SPA output voltage spectrum, it is useful to compare the simulation related histograms shown in Figures $3(a),(c),(e)$ and $4(a),(c),(e)$ with each other. As follows from this comparison, in the histograms in Figure 4, which were obtained using simulation model with transistors whose switching times are less as much as 
two times as compared with the transistors related to the histograms in Figure 3 (the rise time of "slow" transistor is $1.5 \mathrm{~ns}$, and the fall time is $2 \mathrm{~ns}$ ), the harmonic distortions amplitudes are less by an average of $20 \mathrm{~dB}$.

While retaining an acceptable simulation accuracy it would be possible to achieve a simulation time reduction due to the joint use of an approximate analytical method for calculating the characteristics of the output SPA signal and computer simulation. It emerges because the analytical method with a relatively small time expenditure will make it possible to obtain an approximate solution which is rather close to the required exact value. Totally, this will enable to significantly reduce the total time expenditure at the stage of subsequent approximate solution refinement using a simulation model.

The objective of the paper is to improve the accuracy of the analytical method for calculating the SPA signal spectrum by means of considering non-zero switching times which will result in time expenditures reduce needed for computer simulation.

\section{Model With Non-ZERo Switching Times OF Transistors}

The multiphase SPA output signal in the case of ideal switches is described by expression [6]:

$$
U_{\text {out }}(t, N, q)=\frac{\sum_{m=1}^{N}\left\{1+\operatorname{sign}\left[s_{E}(t)-p(t, m, q)\right]\right\}}{2 N},
$$

where $s_{E}(t)$ - a envelope signal, $p(t, m, q)$ - sawtooth reference voltage, $t$ - time, $m$ - phase number, $N$ - total amount of phases in SPA, $q$ - ratio of reference voltage frequency to the maximum frequency in $s_{E}(t)$ spectrum.

In order to take into account the non-zero switching times of transistors, it is necessary to replace the classic function sign $(x)$, which changes its value instantly when the sign of the parameter $x$ changes, to the modified function $\operatorname{sign}^{*}(x)$, in which the transition interval of $x_{\tau}$ duration is set. If the inequality $|x| \geq x_{\tau}$ is true, the $\operatorname{sign}^{*}(x)$ function accepts value \pm 1 . In case $-x_{\tau}<x<x_{\tau}, \operatorname{sign}^{*}(x)=f(x)$, where $f(x)$ is an auxiliary function by means of which the required law of voltage change can be specified at time intervals, when transistors switching occurs. The Figure 2 illustrates the behavior of the auxiliary function $f(x)$ corresponding to the typical cases of linear $(a)$ and exponential $(b)$ simulation of voltage drop on the transistor at the switching time interval.

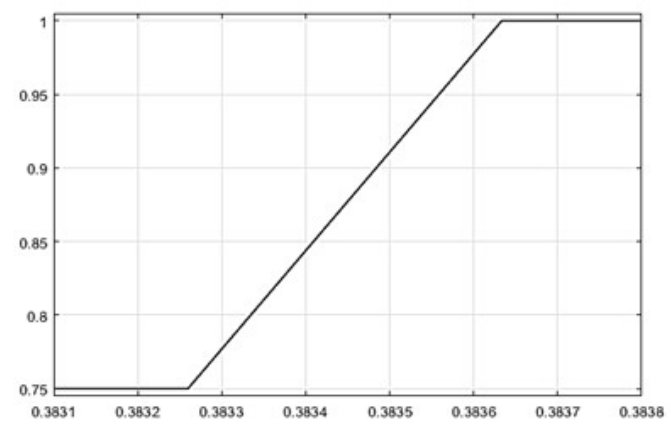

(a)

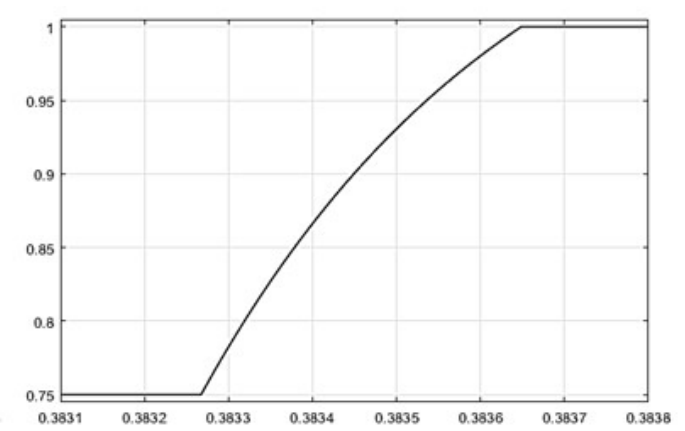

(b)

Figure 2. The switching time interval described by the auxiliary function $f(x)$ for linear $(a)$ and exponential $(b)$ approximation 


\section{RESULTS}

With the use of this method the SPA output signal spectrum was calculated for the cases when the voltage drop across transistors at the stage of their commutation is approximated either linearly or exponentially.

The SPA output signal spectral diagrams corresponding to the linear approximation of the switching times (light-tone histogram) are shown in Figures $3(b),(d),(f)$ and $4(b),(d),(f)$.

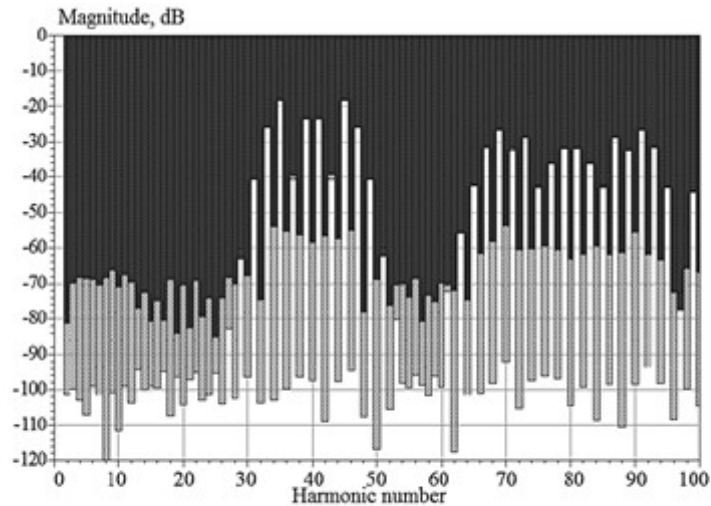

(a)

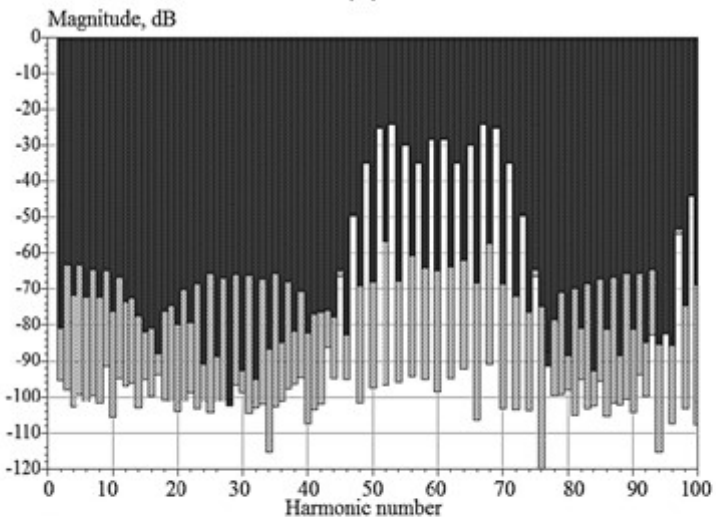

(c)

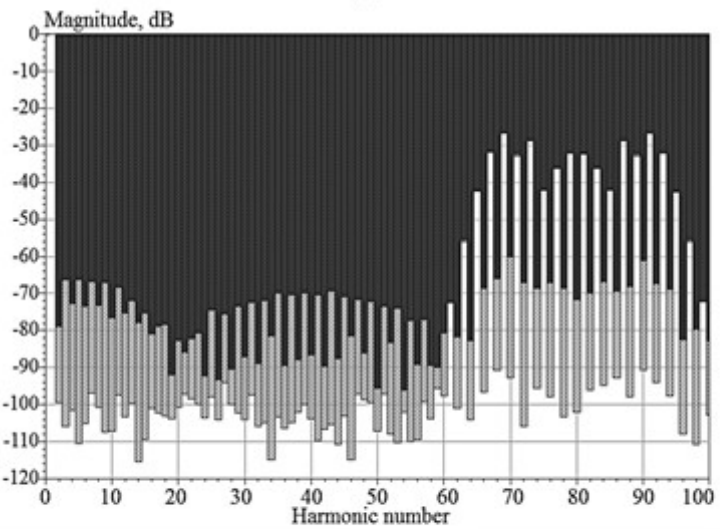

(e)

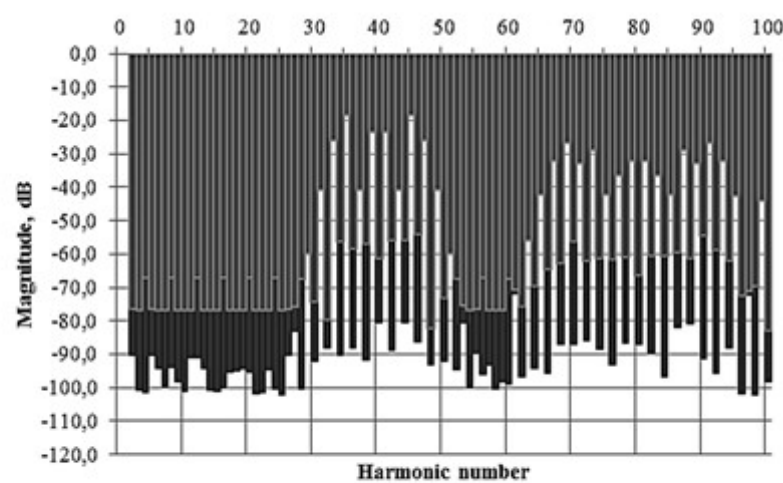

(b)

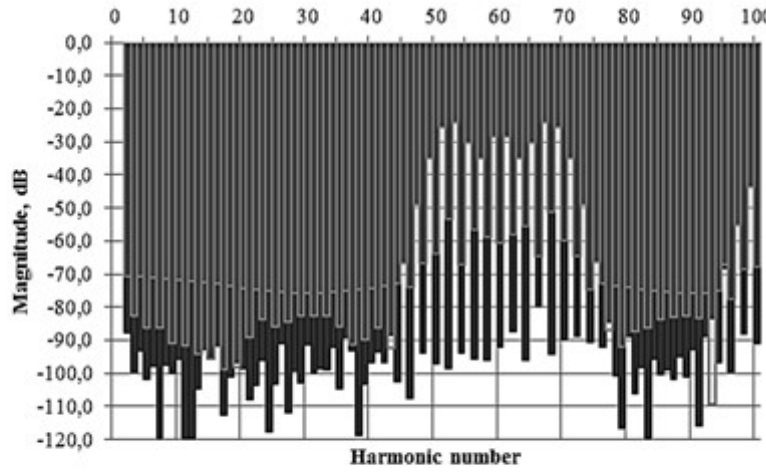

(d)

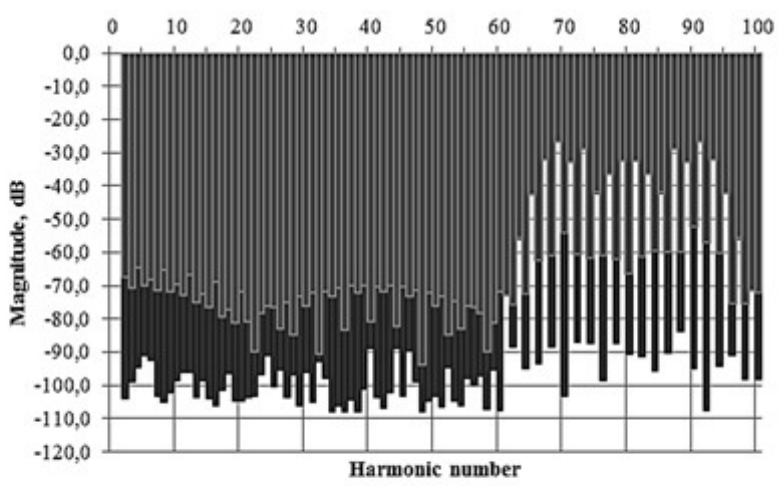

(f)

Figure 3. Spectral diagrams of the SPA output signal for "slow" transistors: $(a),(c)$ and $(e)$ - SPA simulation model (dark tone) and an approximate model with zero switching times of the transistors (light tone); $(b)$, (d) and $(f)$ - the modified model with linear switching times approximation (light tone) and the approximate model with zero switching times(dark tone) 
Pergushev A. O., Sorotsky V. A.

"A time-reduced method for calculation distortions in envelope tracking power amplifiers"

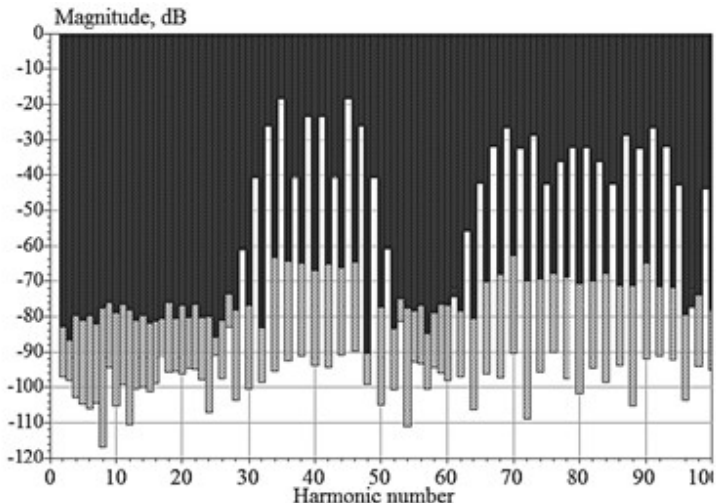

(a)

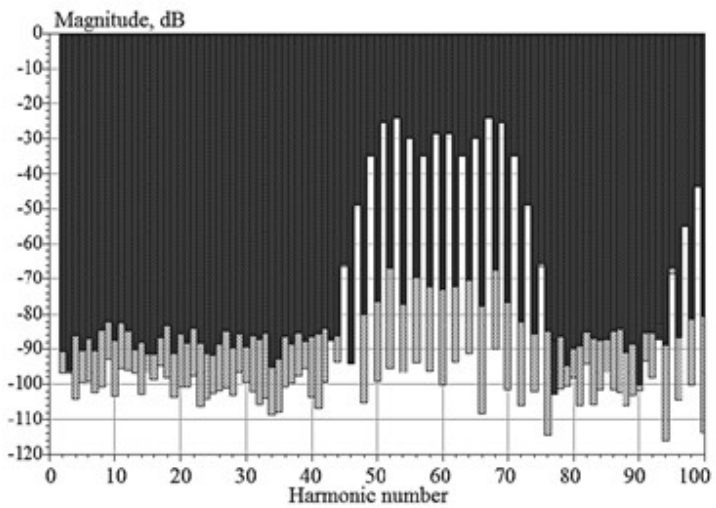

(c)

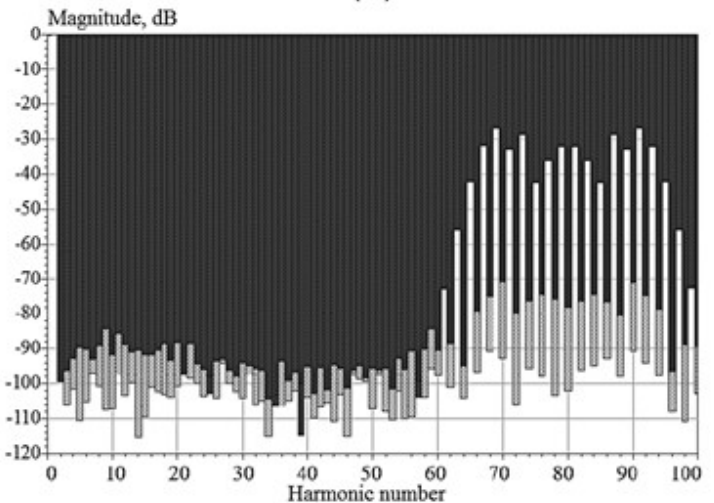

(e)

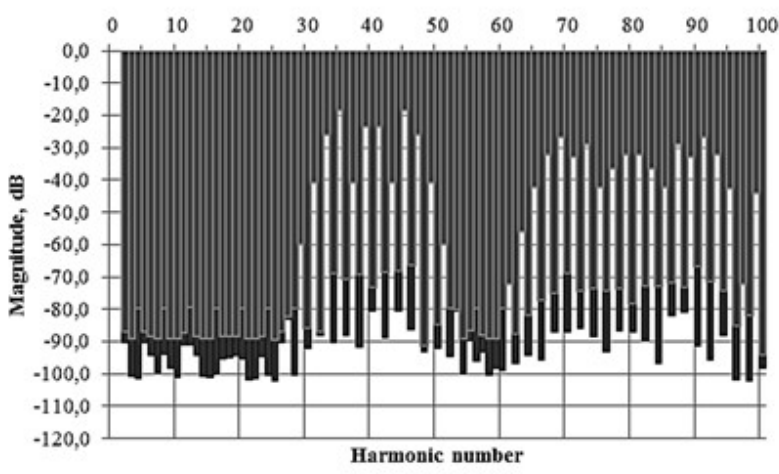

(b)

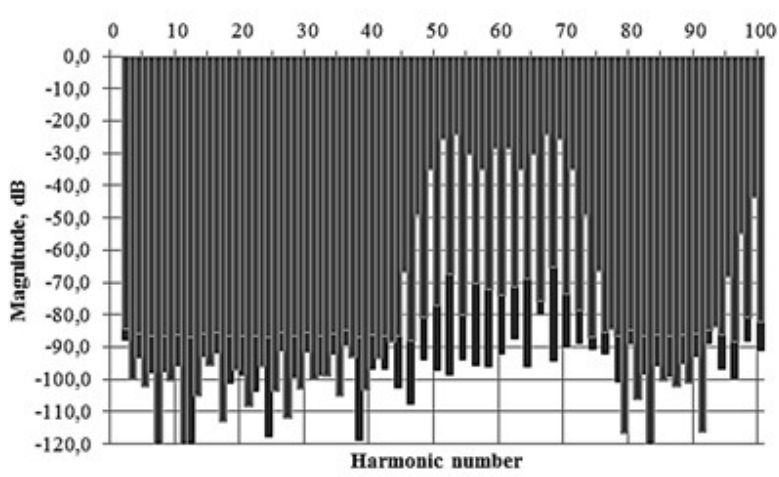

(d)

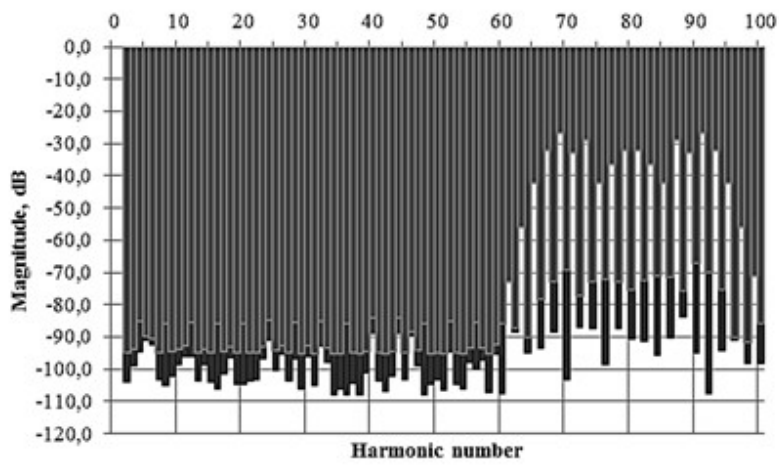

(f)

Figure 4. Spectral diagrams of the SPA output signal for "fast" transistors: $(a),(c)$ and $(e)$ - SPA simulation model (dark tone) and an approximate model with zero switching times of the transistors (light tone); $(b)$, $(d)$ and $(f)$ - the modified model with linear switching times approximation (light tone) and the approximate model with zero switching times(dark tone)

Here, to make comparison more convenient, diagrams corresponding to inertia-free transistors (dark tone histograms), which were calculated with the help of (1), are given. Comparing these results with the results of simulation, it is easy to see that the error in determining the amplitudes of the harmonics has decreased from $30 \ldots 35 \mathrm{~dB}$ to $1 \ldots 6 \mathrm{~dB}$.

Analysis of the results corresponding to linear and exponential voltage drop approximations revealed that the difference in the amplitudes of the harmonics does not exceed $1 \mathrm{~dB}$. 


\section{CONCLUSION}

The proposed method reduces the error in determining the harmonic distortion amplitudes in comparison with (1) by $4 \ldots .5$ times and it should be taken into account that it requires as much as $300 \ldots 800$ times less simulation time to determine SPA output signal spectrum. It can especially be useful for optimization procedures and, as it was shown, a linear function $f(x)$ will suit in most cases.

\section{REFERENCES}

1. Vasic, M., Cheng, P., Garcia, O., Oliver, J. A., Alou, P., Cobos, J. A., ... Ortega-Gonzalez, F. J. (2016). The design of a multilevel envelope tracking amplifier based on a multiphase buck converter. IEEE Transactions on Power Electronics, 31(6), 4611-4627. doi: 10.1109/tpel.2015.2476517.

2. Jin, Q., \& Ruan, X. (2013). Switch-linear hybrid envelope-tracking power supply with multilevel structure. In: IECON 2013 - 39th Annual Conference of the IEEE Industrial Electronics Society (pp. 1325-1330). Vienna, Austria : IEEE. doi: 10.1109/iecon.2013.6699324.

3. Sankman, J., Song, M. K., \& Ma, D. (2016). Switching-converter-only multiphase envelope modulator with slew rate enhancer for LTE power amplifier applications. IEEE Transactions on Power Electronics, 31(1), 817-826. doi: 10.1109/tpel.2015.2406710.

4. Sung, S., Hong, S., Bang, J., Paek, J., Lee, S., Cho, T. B., \& Cho, G. (2016). Envelope modulator for 1.5-W 10-MHz LTE PA without AC coupling capacitor achieving $86.5 \%$ peak efficiency. IEEE Transactions on Power Electronics, 31(12), 8282-8292. doi: 10.1109/tpel.2016.2519243.

5. Meng, F., Zhu, X.-W., Jin, Q., Wang, Y., \& Ruan, X. (2015). Digitally-controlled high-efficiency series form switch-linear hybrid supply modulator for envelope tracking power amplifiers. 2015 Asia-Pacific Microwave Conference (APMC), 3 (pp. 1-3). Nanjing, China : IEEE. doi: 10.1109/apmc.2015.7413431.

6. Kobzev, A. V., Mikhalchenko, G. Y., \& Muzychko, N. M. (1990). Modulyatsionnyye istochniki pitaniya REA [Modulated power supplies for radio electronic devices]. Tomsk, Russia : Radio and communication (in Russian). 